AC 2011-389: WIRELESS TECHNOLOGIES IN INDUSTRIAL AUTOMATION SYSTEMS

Javad Shakib, DeVry University, Pomona

Dr. Mohammad Rafiq Muqri, DeVry University, Pomona 


\section{Wireless Technologies in Industrial Automation Systems}

Automatic control has played a vital role in the advance of engineering and science and it has become an important and integral part of modern manufacturing and industrial processes. One of the main ingredients of automation is undoubtedly control, which means information being collected, processed and delivered back to each actuator.

Although the use of wireline-based field area networks is dominant, wireless technology offers a range of incentives in a number of application areas. In industrial automation, for example, wireless device (sensor/actuator) networks can provide the support for mobile robots operation, monitoring and control of hazardous and difficult-to-access equipment.

This paper attempts to discuss the benefits of the extensive use of wireless technology in process automation. Then a comprehensive overview of the commercial-of-the-shelf wireless technologies including IEEE 802.15.1/Bluetooth, IEEE 802.15.4/ZigBee, and IEEE 802.11 variants is presented and evaluated. The suitability of these technologies for industrial deployment is also evaluated. Furthermore application scenarios and environments, coexistence of wireless technologies, and implementation of wireless fieldbus services are discussed.

The most suitable wireless communication system to operate effectively in an industrial/factory floor environment with improved channel quality, robustness and loss-tolerance applications are introduced. Moreover the next step is towards a unified communication interface and the integration methods are presented as well.

\section{Introduction}

The convenience of true mobility offered by wireless connectivity is the main factor behind widespread acceptance of wireless technologies.

This paper presents the development and application of a practical wireless systems taught in a junior level to electronics and computer technology engineering students.

This paper attempts to explain how this learning and teaching module is instrumental in progressive learning for students by reviewing and combining different parts of communication and industrial automation. It also will serve as an innovative way to expose technology students to these topics while having fun learning the Industrial Applications.

The global system for mobile communication (GSM), a 3G and 4G cellular system designed mainly for mobile telephony, currently has more than one billion users worldwide. Systems like GSM or the third-generation universal mobile telecommunication system (UMTS) nevertheless require extensive infrastructure. The commercial and industrial deployment of systems that function on a smaller scale and do not require costly frequency licensing or infrastructure has 
become more appealing; such systems include wireless personal area networks (WPANs) and wireless local area networks (WLANs).

As a consequence, the Bluetooth and IEEE 802.11 technologies and the newly emerging ZigBee have received a significant amount of public and scientific attention. Bluetooth, like ZigBee, is a typical WPAN representative that is inexpensive, consumes little power, is small in size, and supports voice and data services. The different IEEE 802.11 variants are WLAN representatives that provide comparably high user data rates at the cost of higher battery power consumption. ZigBee is limited to small data rates, but consumes very little power. With their original purposes fulfilled, new areas of deployment are in the area of industrial applications ${ }^{3}$. IEEE 802.11 is considered for seamless coverage of complete cities ${ }^{3,4}$.

Depending on the exact application, users of these wireless technologies have certain expectations concerning the quality of the systems. The application requirements have to be considered carefully in order to be able to choose the most suitable technology. The main criteria are generally throughput, delay, and reliability. In addition, cost, power consumption, security, and, availability can be important issues. Due to a number of issues including radio interference, adverse weather conditions, and inadequate range limits, hard quality-of-service (QoS) guaranty for throughput cannot be provided by wireless systems. In industrial environments, the radio conditions can be especially difficult because metal walls have a significant impact on the transmission. Metal shields causing more reflections during radio transmission is well known. Security dependent systems requiring stringent data rate throughput within strict time window conditions should not be wireless.

In addition to the parameters discussed above - factors like unit density, traffic demand, mobility, environmental changes during deployment, interference, frequency range, etc., determine how well a technology satisfies the requirements. Thus, both the individual link performance and the overall network capacity should be optimized.

One of the main ingredients of automation is undoubtedly control, which means information is being collected, processed and delivered back to each actuator. In this context, telematics plays a key role in the automation industry. To manage at best the requirements of diverse environment, several industrial communication standards have mushroomed all over the world. To improve the portability and the interoperability of these standards, a number of attempts are still running to unify and consolidate them into fewer and widely used approaches. In this process, Ethernet (IEEE 802.3), the well known standard of wireless network communication, seems to play a vital role, thanks to the capability to integrate the heterogeneous traffics of office and industry automation over a single infrastructure. Furthermore, the idea of applying wireless to automated industrial processes is gaining ground. This raises some important questions, such as:

- What are the benefits of the extensive use of wireless technology in process automation?

- What is the best wireless technology for industrial automation?

- Is wireless the suitable next step towards a unified communication interface?

This paper tries to answer these and other questions by venturing new perspectives, brainstorming rich ideas and attempts to draw conclusions. 


\section{Wireless in the industry: benefits, threats and true restrictions}

When considering the introduction of wireless technology into manufacturing plants, different aspects must be considered:

1. Costs. The first and prime reason justifying wireless deployment is always substantial cost saving due to wire replacement; the industrial case is particularly critical due to the high cost of industrial wiring.

2. Resiliency and Safety. The impact of a link failure event over system safety must be minimized: wireless is vulnerable to noise, temporary interferences, fading. A receiver can be "jammed" quite easily. Usually, these are the first objections to wireless: there are several possible solutions preventing such problems and, wires can be cut (and hard to repair) and wired devices (switches, hubs, repeaters) are prone to malfunction!

3. Priority. Safety requirements involve the use of a protocol which is reliable and offers realtime guarantees for the most important signals. Not all the protocols confirm to this criteria. Consider a simplistic safe approach which interrupts processes whenever messages get lost, this may lead to many interruptions reducing your process efficiency and throughput.

4. Security. Another threat concerns vulnerability of wireless under stringent conditions. A number of solutions exist or are in progress to improve the privacy and security of wireless transmissions.

5. Mobility. Wireless means mobility. Freedom from wires brings several benefits: you can move around your plant without disrupting connectivity; in case of frequent reconfiguration of your plant involving assembly lines, you do not have to deal with cable bonds. In most cases, an industrial application requires free nomadic approach than a true mobile solution: this means that you work in quasi-static scenarios on which wireless is particularly effective.

6. Scalability. Intuitively, a wireless solution is more efficient if it allows for an increase in the number of users connected to the same device (overcoming the paradigm of a point-to-point connection), number of active networks, and capability to automatically configure. This will be further discussed in next section.

7. Protocols Inter-operation. Several different industrial communication standards compete and cannot inter-work with each other. A wireless protocol can behave as a bridging protocol among them.

8. Fabric-to-Office Integration. A wireless protocol can efficiently transport office-related and internet-oriented traffic. This would allow to carry on the evolution started by industrial Ethernet, network maintenance costs optimization and always-on connection to the office. The integration with the office (the so called "global networking") enables new perspective, in value added industrial management (automated asset management, supply chain management, customer relationship management).

9. Dynamic Chain Configuration. On the contrary, fabric-to-office integration enables to draw an improved production environment, which is flexible and dynamically re-configurable according to highly differentiated customer requests recalling data (for instance orders) stored on other systems.

Multiple access wireless means that not only a single operator can connect several units at the same time, but several operators can also monitor and collect alarms from the same unit. 
Furthermore, several units can coordinate at run time; this implements a general purpose scenario which can be called "multipoint wireless" (including but not limited to machine-to-machine, man to- machine or machine-to-man data communication). Moreover, with a multiple-access capability, additional data, such as video sequences or sensor measurements, could be contemporarily collected. This would significantly improve early fault detection and diagnosis: monitoring could get more efficient and even a large plant can be potentially monitored from a single location. This means enhanced channel flexibility but would require a large capacity (that is a broadband wireless technology) and a highly flexible channel capable of differentiating among different data profiles (that is a differentiated or prioritized technology).

\section{Wireless characteristics}

The previous sections have argued that the challenge of wireless deployment in an industrial setting is very demanding in terms of various issues and stringent requirements as such the selection of the right wireless technology is crucial.

A plethora of wireless technologies exist, spanning from narrowband to broadband, from shortto long-range, licensed and unlicensed, standard or proprietary, application-dependant and general purpose, point-to-point or multiple-access. Due to strict requirements of automation scenarios a suitable wireless solution must fulfill at least some of these important characteristics:

- resilient and reliable

- immune to interference from other radio sources

- multiple-access

- data- and Internet-oriented

- priority capable

- broadband

- scalable

- secure

\section{Which technologies?}

The burning question is then: "Which wireless technology?"

Let's recall the rational towards the final decision:

- The desired wireless solution should be based on a standard. This is fundamental to avoid the complexity of a multitude of proprietary incompatible solutions.

- The range of communication is expected to be in personal (WPAN) or local range (WLAN). Wide area (WWAN) and metropolitan area networks (WMAN) seem to be less suitable for several reasons. First, they involve a strategic partnership with some public (licensed) telecom operator. Second, they involve higher costs (the costs due to the public services and the higher costs of the devices). Third, they are quite rigid in their configuration with a star topology and a central base station. Furthermore, the bandwidth (with currently deployed solutions) is not large enough.

- These points are sufficient do discard WMAN, GSM, UMTS and all proprietary solutions. Furthermore: 
- RFID cannot be applied to a critical safe solution. It is a low cost and low power solution which offers limited payload and simple functionality. It is suitable to keep track of goods and recognize them throughout an industrial process, but not to pilot an industrial plant and to guarantee flexible and huge data exchange. It can work as an additional collateral technology, not as the main one.

- Bluetooth $(2.4 \mathrm{GHz})$ played an interesting role in the past for WPANs: it offers a good compromise between data rate (about $1 \mathrm{Mbps}$ ) and range (about $10 \mathrm{~m}$ or $100 \mathrm{~m}$ with lower data rates) but this trade-off seems to be its main limit: UWB seems to be preferred for higher data rates and Zigbee preferred due to low power consumption and longer battery life.

- Ultra-Wide Band - UWB (3.1-10.7 GHz) is very promising: this technology can handle the bandwidth required to transport multiple real-time services (including audio and videos!) up to $450 \mathrm{Mbps}$ in short range. It is being investigated as replacement of USB and IEEE Firewire (1394). UWB provides a new paradigm with RF energy spread over several GHz and at such low power density appear to be harmless noise. This has been studied but not ratified by IEEE yet in the 802.15 .3 family.

- Zigbee $(2.4 \mathrm{GHz})$ is focused on ensuring a very long primary battery life rather than high data rate (low duty cycle). Consequently, nodes are able to remain quiescent for long periods without communications. Based on high-density star and mesh networks and using a DSSS approach (see below), this technology can guarantee low latency but only to low throughput data (about 20-200 Kbps).

- HyperLAN (2.4 GHz) is an effective technology but an European standard which is losing ground due to cost considerations.

- Consequently, only WLAN survives the required screening process. In next section (which WLAN) it is shown that it fulfills all the requirements previously mentioned and that a WLAN solution in particular seems suitable for the industrial implementation.

\section{Which WLAN?}

WLAN (or Wi-Fi) is based on a set of well-known standards (the 802.11 family) developed by the IEEE organization: most of them have already been ratified, while new developments, mostly backward compatible, are still being finalized to further improve them. This is a first reason supporting WLANs.

Second, WLAN (or Wi-Fi) was designed as a wireless extension of Ethernet, so it can simply guarantee a natural interworking with internet-like data. This would enable fabric-to-office integration.

Third, several implementations are already available and quite cheap. Furthermore, open source tools and drivers simplify the development of custom solutions.

Fourth, Wi-Fi bandwidth is suitable for data rates required by the industrial applications. Available bandwidth ranges from 1 to $11 \mathrm{Mbps}$ in the first releases (802.11 and 802.11b), to 54 Mbps (802.11a and 802.11g) and up to $500 \mathrm{Mbps}$ in the close future (802.11n): each of these standards achieves different performances which will be investigated below. 
Fifth: the reach (about 100m; more with directional antennas) is suitable for industrial applications.

Sixth: the wireless medium is shared among multiple users. Consequently, a MAC (Medium Access Control) protocol allows efficient access to the medium, with low configuration efforts. The simplest solution ("structured mode") foresees a central node (called Access Point - AP) which defines the rules to associate and participate in its network. It would be natural to put an AP in each robot ${ }^{1}$.

Seventh: the WLAN standards use radio frequency range which include portions of the globally allocated Industrial, Scientific, and Medical (ISM) bands in the $2.4 \mathrm{GHz}$ region2, and of unlicensed bands in the $5 \mathrm{GHz}$ region3. This means that no license is required. Although this means complying with regional rules for electro-magnetic emissions and potential external interference, the closed environment typical of a manufacturing plant is a natural shield against these types of interference.

Eighth, Wi-Fi includes solutions to dynamically set the transmission rates (and the redundancy codes) according to the available bit-error rate (BER) and signal-to-noise (SNR) ratios. Transmission is based on "spread spectrum" (SS) techniques, whose common rationale is to expand the digital signal so that the average transmitted spectral power is kept low while maintaining high data rate and reliability performance.

Ninth, security is not a problem. The IEEE standard includes a robust and mature security solution that overcomes the weaknesses of the early "WEP" encryption schemes.

Tenth, power consumption optimization is not critical for wireless robot control applications. One of the most frequent objection to the use of WLAN (for instance in sensor networks) does not hold for robotics.

There are several possible flavors of WLAN in terms of radio transmission schemes, namely: 802.11, 802.11a, 802.11b, 802.11g, and 802.11n. The IEEE 802.11 existing solutions are shortly recalled below.

- 802.11. The first IEEE 802.11 solutions were designed for the 2.4-2.4835 GHz band and used two different spread spectrum techniques: Frequency Hopping (FHSS) and Direct Sequence (DSSS). FHSS is not as complicated as DSSS and uses less energy, but DSSS has longer range. DSSS supports higher data rates from individual physical layers, which is why DSSS was chosen for a higher-rate 802.11 physical layer (802.11b). Both FHSS and DSSS reach $2 \mathrm{Mbps}$ line rate and use approximately $30 \mathrm{MHz}$ for transmission (one third of the band). This limits the number of non overlapping access points within coverage distance to a maximum of three.

- 802.11b specifies a physical modulation using DSSS at data rates of 5.5 and $11 \mathrm{Mbps}$. The frequency bands and number of channels is the same as for the early IEEE 802.11 standard.

- 802.11a operates in the $5 \mathrm{GHz}$ band (5.150-5.350 GHz and 5.725-5.825 GHz). A technique called $\mathrm{OFDM}^{4}$ is used to increase 802.11 data rates to $54 \mathrm{Mbps}$. OFDM 
increases spectral efficiency and allows greater channel throughput. With OFDM, the high-speed data signal is transported via 64 parallel sub-channels within a $20-\mathrm{MHz}$ channel.

- $\quad 802.11 \mathrm{~g}$, similar to $802.11 \mathrm{~b}$, operates in the $2.4 \mathrm{GHz}$ band, and uses one third of the band per channel. 802.11g mimics 802.11a modulation (OFDM) so that it can reach $54 \mathrm{Mbps}$ data rates. The number of non overlapping channels is 12 .

- $802.11 \mathrm{n}$ is a future standard yet to be ratified by the IEEE 802.11 Group. It is supposed to allow for a raw throughput of up to $500 \mathrm{Mbps}$ in the $5 \mathrm{GHz}$ band.

- Consequently, 802.11a is the only standard which all of the following requirements:

- It is already available.

- It provides high data rates: this means more transmission opportunities for critical (automation) data and potential room for other delay-sensitive (multimedia) or highvolume (internet) traffic.

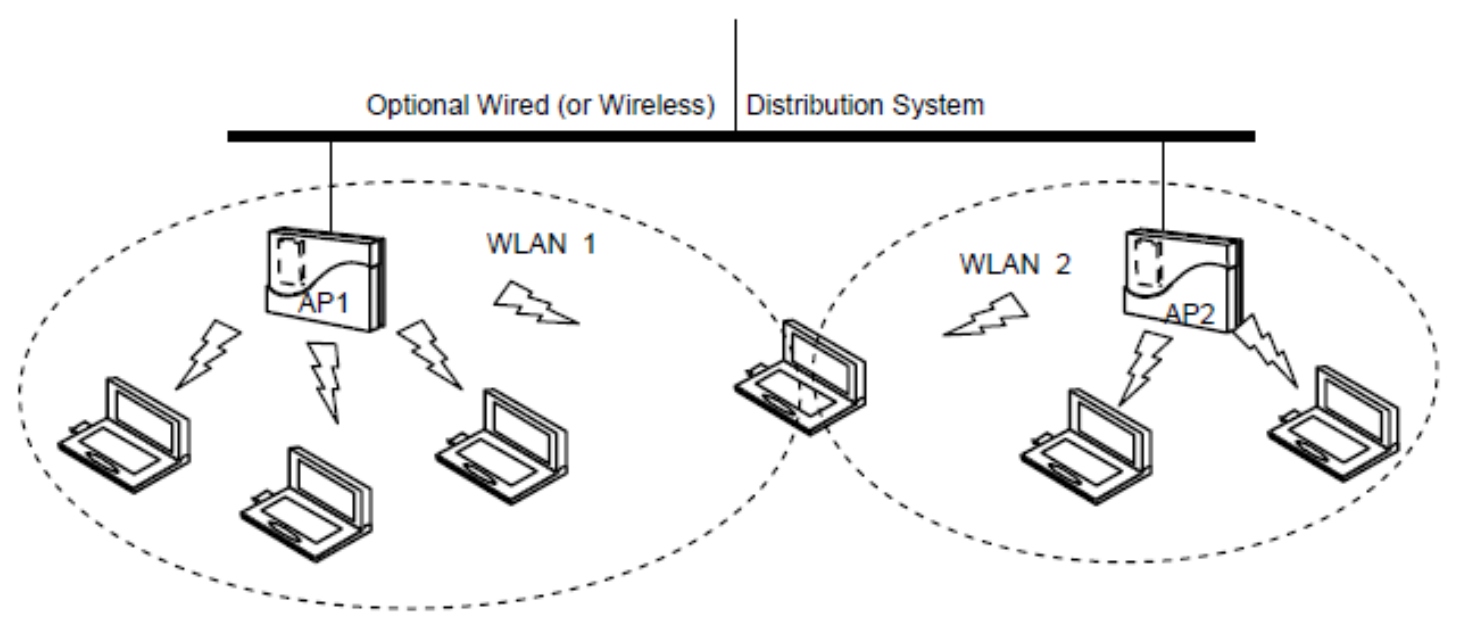

Fig. 1 - Logical architecture of a structured WLAN scenario, with shared wireless medium

- It allows for a high number of non-overlapping channels, which means easier network configuration and opportunity to install several AP able to operate without interfering (operating in physically distinct ranges).

- Operates in the $5 \mathrm{GHz}$ range, which not only allows room for more channels, but is quite prone from undesired interference. In the $2.4 \mathrm{GHz}$ range, $\mathrm{RF}$ interference from other devices, such as the newer cordless phones microwaves ovens, wireless video, Bluetooth devices can reach critical values.

\section{Does it work?}

Although IEEE 802.11a is the most suitable existing solution, it was designed for consumer electronics. Consequently, some modifications must be made to enhance safety and reliability performance. Typical issues which arise when moving to a more critical environment, include:

- addition of resiliency mechanisms to prevent holes in safety due to faulty transmissions (including robot stop)

- potential decrease in the efficiency and availability, mainly due to safety improvement

- indoor radio propagation limitations due to multi-path and undesired scattering 
- interference problems due to other wireless devices (electro-magnetic compatibility issues).

All these issues have been extensively investigated and tested. The results reveal satisfactory performance often beyond expectations. The bandwidth and resilience protocol available in 802.11a are definitely enough to overcome all restrictions.

\section{Summary and Conclusions}

When deciding on a wireless technology to use, you must first clarify the characteristics of the unforeseen application. Do you want to move with or within the network? Do you have mobility within a small range or in a larger area? At what speed do the units move? Do you need access to large databases or just locally?

Is battery life a critical issue? What maximum distance should the wireless link cover? What distance does it cover on average? As a next step, the existing technologies can be viewed for their applicability.

Bluetooth is a quite power-efficient WPAN technology. Like all wireless systems, it cannot provide hard throughput guarantees. Bluetooth is nevertheless quite robust for best-effort traffic in coexistence environments. One hundred Bluetooth piconets can transmit at an average data rate of $(95 \%)$ at $33.2 \mathrm{kbps}$ in an area of $10 \times 20 \mathrm{~m}^{2}$. Fifty fully loaded piconets can transmit at an average, unidirectional transmission rate of $360 \mathrm{kbps}$. Similar results are likely to be achieved on larger factory floors (provided the piconet density is comparable), as the disadvantage of a larger number of units can be outweighed by the more structured and predictable unit location. Of the discussed technologies, Bluetooth is the only one that supports voice in addition to data transmission.

IEEE 802.11 is a WLAN technology that enables higher data rates but is not power consumption friendly. Additionally, in a relatively dense network scenario, the maximum aggregate throughput of 3 times 7.11/30.8 Mbps is likely to be seriously impaired. To aid the WLAN performance on a factory floor, it is thus advisable to take the following two measures: apply means to combat the increased delay spread (in case of IEEE 802.11b) and (for all IEEE 802.11 systems) carefully plan the frequency layout and access point placement.

The newly emerging ZigBee technology has been designed specifically for sensor data and control information at low data rates. ZigBee supports long battery lives. Nothing can be said yet about the robustness and effectiveness of ZigBee. Yet for low-bit-rate applications in industrial environments, ZigBee seems to be a promising approach. The most efficient measure to aid the coexistence of Bluetooth and IEEE $802.11 \mathrm{~b}$ is to use Bluetooth with adaptive frequency hopping. If this is not sufficient, hardware-related improvements and a common scheduler have to be added.

Since IEEE 802.11 is a consumer electronics technology, a number of companies including Comau Robotics, Italy have performed extensive studies, and contributed to the development of new technologies for the effective integration of consumer WiFi in indusrial plants. ${ }^{5}$ 


\section{Bibliography}

1. IEEE Std 802.11, 1999 Edition (R2003) (ISO/IEC 8802-11: 1999) IEEE Standard for Information Technology Telecommunications and Information Exchange between Systems - Local and Metropolitan Area Network - Specific Requirements - Part 11: Wireless LAN Medium Access Control (MAC) and Physical Layer (PHY) Specifications 2. IEEE P802.11REV-ma/D3 Unapproved Draft Standard (This document reflects the combining of the 2003 Edition of 802.11 plus the $802.11 \mathrm{~g}, 802.11 \mathrm{~h}, 802.11 \mathrm{i}$ and $802.11 \mathrm{j}$ Amendments).

3. A. Willig, K. Matheus, and A. Wolisz, "Wireless Technology in Industrial Networks", Proceedings of the IEEE, vol. 93, no. 6, pp. 1130-1151, June 2005.

4. A. Willig, M. Kubisch, C. Hoene, and A. Wolisz, "Measurements of a Wireless Link in an Industrial Environment using an IEEE 802.11-Compliant Physical Layer", IEEE Transactions on Industrial Electronics, vol. 43, no. 6, pp. 1265-1282, December 2002.

5. Brevi D., Pilosu, L., Fileppo F., Scopigno R., "Viability and Guidelines for the Effective Integration of Consumer WiFi in Industrial Plants", Ultra Modern telecommunications and Control Systems and Workshops (ICUMT). DOI: 10.1109/ICUMT.2010.5676632 